\title{
Importance of Mitral Subvalvular Apparatus in Terms of Cardiac Energetics and Systolic Mechanics in the Ejecting Canine Heart
}

\author{
Kwok L. Yun, Marek A. Niczyporuk, George E. Sarris, James I. Fann, and D. Craig Miller \\ With the technical assistance of Geraldine C. Derby and Cynthia E. Handen \\ Department of Cardiovascular Surgery, Stanford University School of Medicine, Stanford, California 94305
}

\section{Abstract}

To assess the importance of the intact mitral subvalvular apparatus for left ventricular (LV) energetics, data from nine openchest ejecting canine hearts were analyzed using piezoelectric crystals to measure $L V$ volume. After mitral valve replacement with preservation of all chordae tendineae, baseline $\mathrm{LV}$ function was assessed during transient caval occlusion: A quadratic fit of the LV end-systolic pressure-volume data was used to determine the curvilinear end-systolic pressure-volume relationship (ESPVR). All chordae were then divided with exteriorized snares. Reassessment revealed deterioration of global LV pump function: $(a)$ the coefficient of nonlinearity, decreased (less negative) by $90 \%(P=0.06)$; (b) slope of the curvilinear $E S P V R$ at the volume axis intercept, decreased by $75 \%(P$ $=0.01$ ); and $V_{100}$, end-systolic volume at $100 \mathrm{mmHg}$ end-systolic pressure, increased by $42 \%(P<0.02)$. Similarly, preload recruitable stroke work fell significantly $(-14 \%)$ and $V_{w 1,000}$ (end-diastolic volume [EDV] at stroke work [SW] of 1,000 $\mathrm{mmHg} \cdot \mathrm{ml})$ rose by $17 \%(P<0.04)$. With respect to $\mathrm{LV}$ energetics, the total mechanical energy generated by the ventricle decreased, as indicated by a decline in the slope of the pressure volume area $(P V A)-E D V$ relationship $(120 \pm 13$ [mean $\pm S D]$ vs. $105 \pm 13 \mathrm{mmHg}, P<0.001)$. Additionally, comparison of $\mathrm{LV}$ $S W$ and $P V A$ from single beats with matched $E D V$ showed that the efficiency of converting mechanical energy to external work $(S W / P V A)$ declined by $14 \%(0.65 \pm 0.13$ vs. $0.56 \pm 0.08, P$ $<0.03)$ after chordal division. While effective systemic arterial elastance, $E_{a}$, also fell significantly $(P=0.03)$ after the chordae were severed, the $E_{\mathrm{a}} / E_{\mathrm{es}}$ ratio $\left(E_{\mathrm{es}}=\right.$ slope of the linear ESPVR) increased by $124 \%(0.91 \pm 0.53$ vs. $2.04 \pm 0.87, P$ $=0.001)$ due to a proportionally greater decline in $E_{\mathrm{es}}$. This indicates a mismatch in ventriculo-arterial interaction, deviating from that required for maximal external output (viz., $E_{a} / E_{e}$ $=1$ ). These adverse effects of chordal division may be related to the observed changes in LV geometry (i.e., eccentricity). We conclude that the intact mitral subvalvular apparatus is important in optimizing $L V$ energetics and ventriculo-vascular coupling in addition to the enhancement of $\mathrm{LV}$ systolic performance. (J. Clin. Invest. 1991. 87:247-254.) Key words: chordae tendinae $\bullet \mathbf{L V}$ function $\cdot$ ventriculo-vascular interaction

Address reprint requests to Dr. D. Craig Miller, Department of Cardiovascular Surgery, Cardiovascular Research Center, Stanford University Medical Center, Stanford, CA 94305. 1990

Received for publication 29 March 1990 and in revised form 26 July

J. Clin. Invest.

(c) The American Society for Clinical Investigation, Inc.

0021-9738/91/01/0247/08 $\$ 2.00$

Volume 87, January 1991, 247-254

\section{Introduction}

Mitral papillary-annular continuity has been hypothesized to be important for optimal global left ventricular (LV) ${ }^{1}$ systolic performance, a concept we have previously termed "valvularventricular interaction" (1). In isovolumic experimental preparations, it has been shown that chordal division results in deterioration of LV systolic mechanics (1-6), which can be restored by reattachment of the mitral chordae (5). The anterior and posterior leaflet chordae have similar, but additive, contributions to LV systolic elastance (4). More recently, the importance of the integrity of the mitral subvalvular apparatus for LV pump function has been confirmed in in situ ejecting canine hearts using relatively load-insensitive indexes of LV systolic function (7); chordal severing resulted in a $72 \%$ decline in $E_{\text {es }}$, slope of the linear end-systolic pressure-volume relationship (ESPVR). The extrapolated volume intercept $\left(V_{0}\right)$ also fell significantly, but was in the (nonphysiologic) negative range $(-16.6 \pm 15.3 \mathrm{ml})$ after chordal disruption. The possibility that ESPVR was nonlinear outside the physiologic pressure range examined or whether there was an actual change in the curvilinearity of the ESPVR is unknown. Moreover, the implications of this deterioration in systolic mechanics in terms of $\mathrm{LV}$ energetics and efficiency were not examined in the setting of altered LV geometry (vs. change in contractile state). We therefore assessed the effects of chordal division on $\mathrm{LV}$ myocardial energetics, mechanical energy (pressure volume area) to external pressure-volume work efficiency, and systolic pump function using curvilinear ESPVR analysis. It is important that ventriculo-arterial coupling, which ultimately determines the efficiency of energy transfer to the systemic circulation, was examined before and after chordal severing, since LV systolic function may not necessarily be related to ventriculo-vascular interaction (8).

\section{Methods}

Surgical preparation. This surgical preparation has been described in detail previously (7). Briefly, nine adult, mongrel dogs (25-30 kg) were anesthetized with thiopental $(25 \mathrm{mg} / \mathrm{kg})$ and maintained with $0.5-1 \%$
1. Abbreviations used in this paper: $E_{\mathrm{a}}$, effective systemic arterial elastance; $E_{\mathrm{es}}$, slope of the linear ESPVR; $E D D$, end-diastolic volume; $E D P$, end-diastolic pressure; $E D V$, end-diastolic volume; EED, endejection diameter; EM, electromagnetic; ESPVR, end-systolic pressure-volume relationship; $L V$, left ventricular; $M E P$, mean ejection pressure; \%dD, fractional diameter shortening; $P_{\text {es }}$, end-systolic pressure; PRSW, preload recruitable stroke work; PVA, pressure-volume area; $S V$, stroke volume; $S V_{\text {sono }}, S V$ as determined by sonomicrometry; $S W$, stroke work; $V_{\text {es }}$, end-systolic volume; $V_{0}$, volume axis intercept; $V_{\text {pva }}$, preload recruitable PVA volume intercept. 
halothane. The heart was exposed via a left lateral thoracotomy, and sets of piezoelectric crystals were implanted to allow continuous measurement of three orthogonal LV dimensions (base-apex $\left[D_{\mathrm{BA}}\right]$, anterior-posterior $\left[D_{\mathrm{AP}}\right]$, and septal-lateral $\left.\left[D_{\mathrm{SL}}\right]\right)$. A silastic occluder was placed around the inferior vena cava to alter preload abruptly. On cardiopulmonary bypass the mitral valve was exposed through a left atriotomy, and all chordae were snared with 0-Flexon wire sutures and exteriorized through plastic tubing that was secured to the epicardium. A 25-mm porcine bioprosthesis was then sutured to the mitral annulus with complete preservation of the subvalvular apparatus. The atriotomy was closed, and the animal weaned from cardiopulmonary bypass. A micromanometer-tipped catheter was inserted into the LV cavity via the apex, and a fluid-filled catheter was inserted into the left atrium to guide volume replacement. An electromagnetic (EM) flow probe was placed around the ascending aorta to measure instantaneous flow. This report is based on further analysis of data derived from the same animals reported earlier by Sarris et al. (7).

Experimental protocol. After $1 \mathrm{~h}$ of reperfusion and stabilization of arterial pressure and cardiac output, baseline hemodynamics and dimensional data were obtained over a physiologic range of peak systolic LV pressures by transient inferior vena cava occlusion (during which the ventilator was temporarily disconnected to minimize respiratory variation). Three baseline measurements were obtained, allowing 3-5 min between data acquisition runs for steady-state equilibration. After baseline data were collected, all chordae tendineae were divided by pulling the wire suture snares, and data again collected in triplicate. Warm, fresh, homologous blood was infused to maintain baseline levels of mean arterial pressure.

Analogue data were recorded on a multichannel recorder (model 7758B; Hewlett-Packard Co., Andover, MA) at a paper speed of 25 $\mathrm{mm} / \mathrm{s}$. The following parameters were measured: $\mathrm{LV}$ pressure $(P)$, electronically differentiated LV $d P / d t$, ECG, aortic EM flow, and the three dimensions $D_{\mathrm{BA}}, D_{\mathrm{AP}}$, and $D_{\mathrm{SL}}$. These data were simultaneously digitized at $250 \mathrm{~Hz}$ by a $6942 \mathrm{~A}$ multiprogrammer (Hewlett-Packard), controlled by a computer workstation (series 9,000 , model 216; HewlettPackard), and stored on digital disk drives.

Data analysis. The data were analyzed on a series 9,000 , models 226 and 310 computer workstations using software developed in our laboratory. Premature ventricular beats and the subsequent two postextrasystolic beats were excluded from analysis by an R-R interval sampling algorithm. Instantaneous $\mathrm{LV}$ volume $V(t)$ was calculated by assuming the ventricle to be an ellipsoid and subtracting the directly measured (postmortem) ventricular muscle volume $\left(V_{\text {mus }}\right)(7): V(t)$ $=(\pi / 6)\left(D_{\mathrm{BA}} \cdot D_{\mathrm{AP}} \cdot D_{\mathrm{SL}}\right)-V_{\text {mus }}$. The following variables were calculated for each cardiac cycle: End-diastolic volume $(E D V)$ and end-ejection volume $(E E V)$ (defined as $L V$ volume at peak $+d P / d t$ and peak $-d P / d t$, respectively), stroke volume ( $S V$ ) (computed as $E D V-E E V$ ), cardiac output $(C O)$ (calculated as $S V \cdot$ heart rate), mean ejection pressure $(M E P)$ (determined as the time integral of $\mathrm{LV}$ pressure during ejection defined by the aortic flow signal), and total peripheral resistance (estimated as $M E P / C O$ ). Stroke work $(S W)$ was computed as the time integral of LV pressure and volume for each cardiac cycle: $S W$ $=\int P \cdot d V$. Since the estimation of $\mathrm{LV}$ volume is dependent on chamber geometry, $S V$ derived from sonomicrometric measured LV dimensions for each beat during caval occlusion was compared with that obtained from the time integral of the aortic EM flow signal during ejection, both before and after chordal division, to assure that chordal severing did not alter the relation between true $\mathrm{LV}$ volume and that estimated using the ellipsoid model.

End-systolic pressure $\left(P_{e s}\right)$ and volume $\left(V_{e s}\right)$ were determined using an iterative technique $(9,10)$. To avoid problems with linear extrapolation of a curvilinear relation beyond the data range $(11,12)$, LV volume at an end-systolic pressure of $100 \mathrm{mmHg}, V_{100}$, was calculated. Alternatively, end-systolic data were also fitted to the following parabolic curvilinear model (12): $P_{\mathrm{es}}=a \cdot\left(V_{\mathrm{es}}-V_{0}\right)^{2}+b \cdot\left(V_{\mathrm{es}}-V_{0}\right)$. In this expression, $a$ is the coefficient of curvilinearity, $V_{0}$ is the volume axis intercept, and $b$ is the slope of the ESPVR at $V_{0}$ using a best leastsquared algorithm.
Another load-insensitive measure of contractile state, preload recruitable $S W(P R S W)$, was determined during each caval occlusion by least-squares linear regression relating $S W$ to $E D V(13): S W=M_{\mathrm{w}}$ $\left(E D V-V_{\mathrm{w}}\right)$, where $M_{\mathrm{w}}$ is the slope and $V_{\mathrm{w}}$ is the volume intercept. Again, to avoid extrapolation beyond the range of the observed data, $E D V$ at an $S W$ of $1,000 \mathrm{mmHg} \cdot \mathrm{ml}, V_{\mathrm{w} 1,000}$, was calculated in addition to $V_{\mathrm{w}}$.

The pressure volume area $(P V A)$ (represented as the total area under the nonlinear ESPVR as well as the systolic segment of the pressure-volume trajectory and above the end-diastolic pressure-volume relation curve) was calculated for each beat during caval occlusion by the method of Goto et al. (14). Preload recruitable PVA (PRPVA) was defined as the slope of the least-squares linear regression between $P V A$ and $E D V: P V A=M_{\mathrm{pva}}\left(E D V-V_{\mathrm{pva}}\right)$, where $M_{\mathrm{pva}}$ and $V_{\mathrm{pva}}$ are the slope and volume intercept, respectively. The efficiency of energy transfer from $P V A$ to external mechanical pressure-volume work ( $S W / P V A)$ (15) was calculated during each data acquisition run for beats with matched LV $E D V$. Effective arterial elastance $\left(E_{\mathrm{a}}\right)$, defined as $P_{\mathrm{es}} /$ $\left(E D V-V_{e s}\right)$ by Sunagawa (16), was calculated from the first beat of each caval occlusion during steady-state conditions. The $E_{\mathrm{a}} / E_{\mathrm{es}}$ ratio was then computed, where $E_{\text {es }}$ represents the slope of the linear ESPVR.

To assess the effects of chordal division on LV chamber geometry, the following parameters were calculated for each of the three $L V$ axes studied at a matched LV end-diastolic pressure (EDP): End-diastolic diameter $(E D D)$ and end-ejection diameter $(E E D)$ were defined at the time of $E D V$ and $E E V$, respectively. Percent systolic fractional shortening $(\% \mathrm{dD})$ was calculated as $([E D D-E E D] / E D D) \cdot 100 \%$. Eccentricity (e) was computed as (17): $e=\operatorname{sqrt}\left(D_{\mathrm{BA}^{2}}-D_{\mathrm{AP}^{2}}\right) / D_{\mathrm{BA}}$.

Statistical analysis. All results are expressed as mean \pm 1 SD unless specified otherwise. Mean values for all variables, calculated as the average of all three measurements at baseline and after chordal division, were compared with a two-tailed, paired $t$ test. Comparisons of correlation coefficients were made after Fisher's $z$-transformation. The level of significance was taken as $P \leq 0.05$. A $P$ value $>0.05$ but $\leq 0.10$ was considered to be possibly significant and is stated as such.

\section{Results}

Hemodynamics. Table I summarizes the effects of chordal division on LV hemodynamic parameters. As previously reported (7), dividing the chordae resulted in a small but significant decline in heart rate (from $136 \pm 30$ to $130 \pm 27$ beats/min, $P$ $=0.04$ ). Despite an increase in $E D V$ (from $34 \pm 14$ to $40 \pm 15 \mathrm{ml}$, $P=0.05), S V$ and $C O$ did not change. Compared with baseline values, $M E P$ fell significantly from $107 \pm 11$ to $83 \pm 19 \mathrm{mmHg}$ $(P=0.02)$ after chordal severing, resulting in a possibly significant decline $(P=0.09)$ in calculated total peripheral resistance. Moreover, the relation between $S V$ as determined by sonomicrometry $\left(S V_{\text {sono }}\right)$ and aortic EM flow $\left(S V_{f}\right)$ did not change after severing the chordae tendineae, as illustrated in Fig. 1. At baseline, $S V_{\text {sono }}$ was linearly related to $S V_{\mathrm{f}}$ by the equation: $S V_{\text {sono }}=(0.65 \pm 0.09) \cdot\left(S V_{\mathrm{f}}-[0.17 \pm 2.70]\right)$, with $r$ ranging from 0.956 to 0.998 . After chordal division, this relation remained linear ( $r=0.967-0.994)$ without any significant alteration in either the slope $(0.67 \pm 0.06)$ or the $x$-axis intercept $(0.34 \pm 5.02 \mathrm{ml})$.

Global left ventricular systolic performance. Fig. 2 graphically illustrates the effects of chordal division on the nonlinear ESPVR; data for all animals are summarized in Fig. 3. The nonlinear model fit the observed ESPVR data closely throughout the LV pressure range, with nonlinear correlation coeffcients ranging from 0.962 to 0.998 and 0.943 to 0.998 before and after chordal severing, respectively $(P=$ NS). Although the second order coefficient $a$ varied markedly, the overall value of 
Table I. Hemodynamic Parameters before and after Chordal Division

\begin{tabular}{lcc}
\hline & \multicolumn{2}{c}{ Chordal status } \\
\cline { 2 - 3 } & Intact & Divided \\
\hline Heart rate $\left(\mathrm{min}^{-1}\right)$ & $136 \pm 30$ & $130 \pm 27^{*}$ \\
LV end-diastolic volume $(\mathrm{ml})$ & $34 \pm 14$ & $40 \pm 15^{*}$ \\
LV stroke volume $(\mathrm{ml})$ & $23 \pm 9$ & $25 \pm 9$ \\
Cardiac output (liter/min) & $3.2 \pm 1.4$ & $3.2 \pm 1.4$ \\
Total peripheral resistance $(\mathrm{mmHg}-\mathrm{s} / \mathrm{ml})$ & $2.52 \pm 1.50$ & $2.10 \pm 1.38^{\ddagger}$ \\
Mean LV ejection pressure $(\mathrm{mmHg})$ & $107 \pm 11$ & $83 \pm 19^{8}$ \\
& & \\
\hline
\end{tabular}

Results are expressed as mean $\pm 1 \mathrm{SD}$

Vs. chordae intact: ${ }^{*} P<0.05 ;{ }^{\ddagger} P=0.09 ;{ }^{\S} P=0.02$

$a$ for all runs was $-3.149 \pm 5.755 \mathrm{mmHg} / \mathrm{ml}^{2}$ (95\% confidence limits: -6.216 and $-0.082 \mathrm{mmHg} / \mathrm{ml}^{2}$ ); therefore, the ESPVR was significantly nonlinear, being concave towards the abscissa (LV volume axis). After division of the subvalvular apparatus, the ESPVR became less concave, but this difference was only possibly significant ( $a=-5.7 \pm 17.4$ vs. $-0.6 \pm 0.8 \mathrm{mmHg} / \mathrm{ml}^{2}$; $P=0.064) . V_{0}$ fell $(8.8 \pm 5.7$ vs. $3.4 \pm 7.0 \mathrm{ml} ; P=0.009)$, but remained within the physiologic range. Systolic function, however, as estimated by the slope of the nonlinear ESPVR at $V_{0}, b$, declined markedly by $75 \%$ after chordal division $(46 \pm 34$ vs. $12 \pm 8 \mathrm{mmHg} / \mathrm{ml} ; P=0.01) . V_{100}$ increased significantly by $42 \%$ from $17 \pm 10$ to $25 \pm 15 \mathrm{ml}(P=0.014)$ after the chordae were severed.

As a corollary, changes in PRSW (another load-insensitive measure of LV systolic performance which incorporates both systolic and diastolic functions [12]), were also analyzed (Fig. $3)$. The $S W-E D V$ relationship was linear both before $(r$ $=0.959-0.997)$ and after chordal severing $(r=0.976-0.998)$.

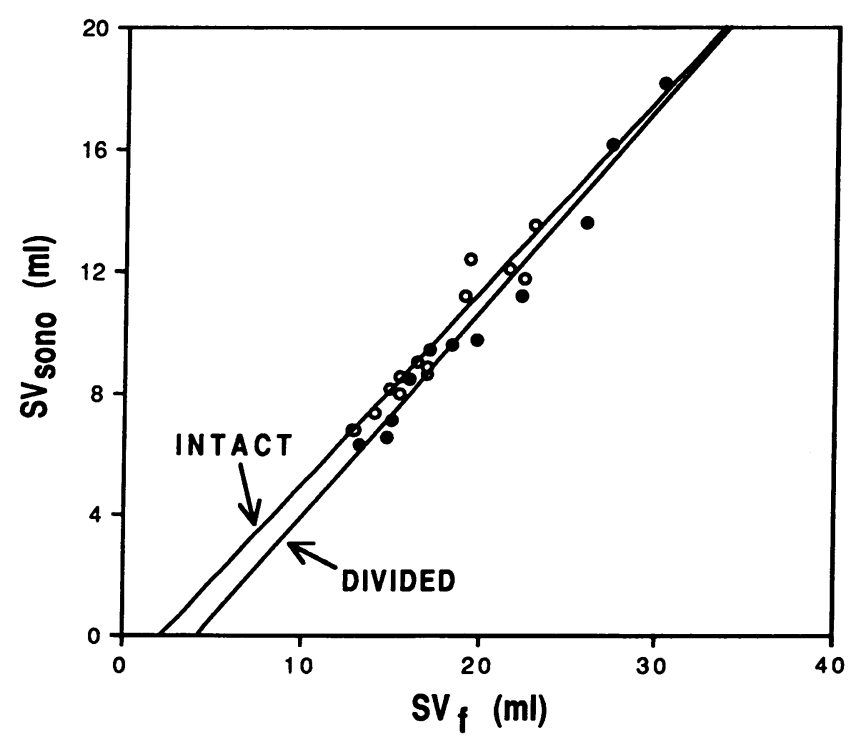

Figure 1. Representative example of the relation between LV stroke volume as derived from sonomicrometric $\mathrm{LV}$ dimension changes $\left(S V_{\text {sono }}\right)$ and that measured directly from the aortic EM flow-signal $\left(S V_{\mathrm{f}}\right)$ during caval occlusion with the chordae tendineae intact (open circles) and divided (closed circles).

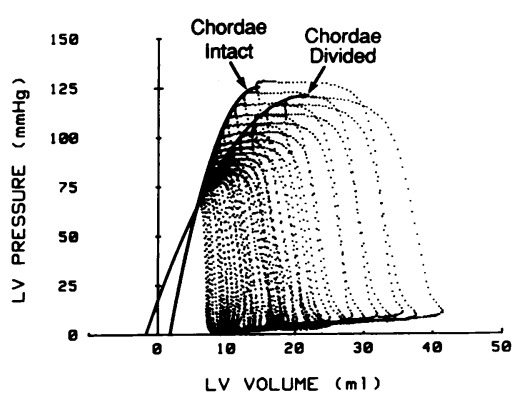

After the chordae were divided, $M_{\mathrm{w}}$ declined by $14 \%$ (81 $\pm 13 \mathrm{vs}$. $69 \pm 16 \mathrm{mmHg} ; P=0.02)$ without consistent changes in the volume intercept, $V_{\mathrm{w}}(13.3 \pm 7.9$ vs. $16.2 \pm 7.2 \mathrm{ml} ; P=0.14)$. As was seen for $V_{100}, V_{\mathrm{w} 1,000}$ increased by $17 \%$ from $27 \pm 8$ to $32 \pm 9$ $\mathrm{ml}(P=0.037)$.

Left ventricular energetics. Fig. 4 illustrates the effect of chordal severing on PRPVA as defined above. In this representative animal, the slope, $M_{\text {pva }}$, fell from 105 to $84 \mathrm{mmHg}$ with minimal reduction in the volume intercept, $V_{\text {pva }}$. In all dogs, the $P V A-E D V$ relationship was highly linear at baseline $(r$ ranged from 0.990 to 0.999 ) and was not affected by chordal severing ( $r=0.995-1.00 ; P=\mathrm{NS})$. Comparison of measurements after chordal division with baseline values for all animals revealed a highly significant $13 \%$ decline in $M_{\text {pva }}(120 \pm 13$ vs. $105 \pm 13 \mathrm{mmHg} ; P<0.001)$ and a $9 \%$ decrease in $V_{\mathrm{pva}}$ $(15.0 \pm 6.6$ vs. $13.7 \pm 6.7 \mathrm{ml} ; P=0.033)$. This implied a reduction in total mechanical work able to be generated at any given LV $E D V$. Furthermore, the $S W / P V A$ ratio at matched $E D V$, reflecting the efficiency of energy transfer from $P V A$ to external mechanical pressure-volume work, also decreased significantly by $14 \%(0.65 \pm 0.13$ vs. $0.56 \pm 0.08 ; P=0.023)$ after chordal disruption (Fig. 5).

When compared with baseline values, steady-state effective arterial elastance, $E_{\mathrm{a}}$, fell significantly by $43 \%$ from $8.6 \pm 5.1$ to $4.9 \pm 1.5 \mathrm{mmHg} / \mathrm{ml}(P=0.031)$ with chordal severing. In terms of ventriculo-arterial coupling, however, the $E_{\mathrm{a}} / E_{\mathrm{es}}$ ratio $\left(E_{\mathrm{es}}\right.$ obtained using linear regression within only a limited LV pressure range) actually increased by over twofold $(0.91 \pm 0.53$ vs. $2.04 \pm 0.87 ; P=0.001$ ), because of a proportionally greater $(-72 \%)$ decline in $E_{\mathrm{es}}$ (Fig. 6). With the chordae intact, the $95 \%$ confidence intervals for $E_{2} / E_{\text {es }}$ ranged from 0.47 to 1.26 , which were not significantly different from unity.

The effects of chordal severing on LV chamber geometry at matched LV EDP are summarized in Table II. After chordal division, the increase in $E D V$ was due to some lengthening of $E D D$ in all three $\mathrm{LV}$ axes, but primarily along the major axis. In contrast, \%dD rose in the anterior-posterior $(P<0.05)$ and possibly the septal-lateral $(P=0.054)$ dimensions. Fractional diameter change during ejection in the base-apex direction was unaltered due to a parallel increase in $E E D(P<0.001)$. As a result, the $L V$ was significantly more eccentric at end-diastole; this was possibly also true at end-ejection $(P=0.067)$.

\section{Discussion}

The concept valvular-ventricular interaction was recently demonstrated in in situ ejecting canine hearts by Sarris et al. (7). 

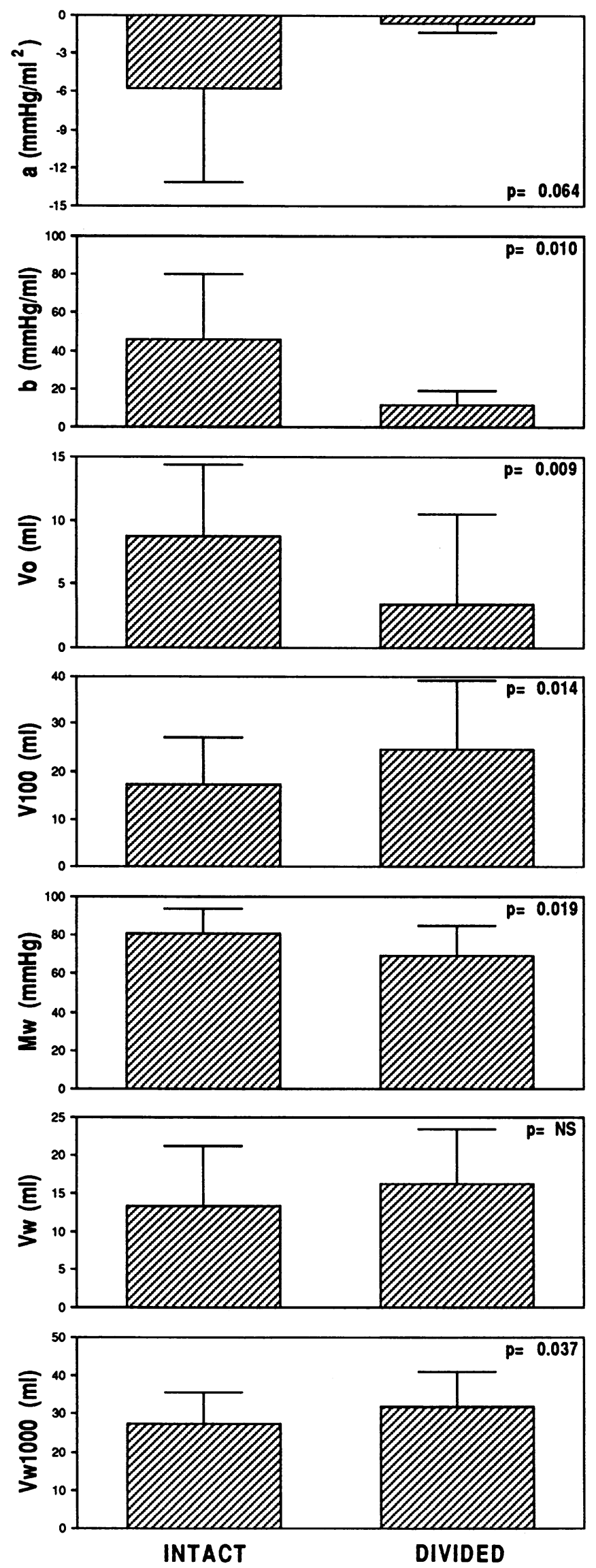

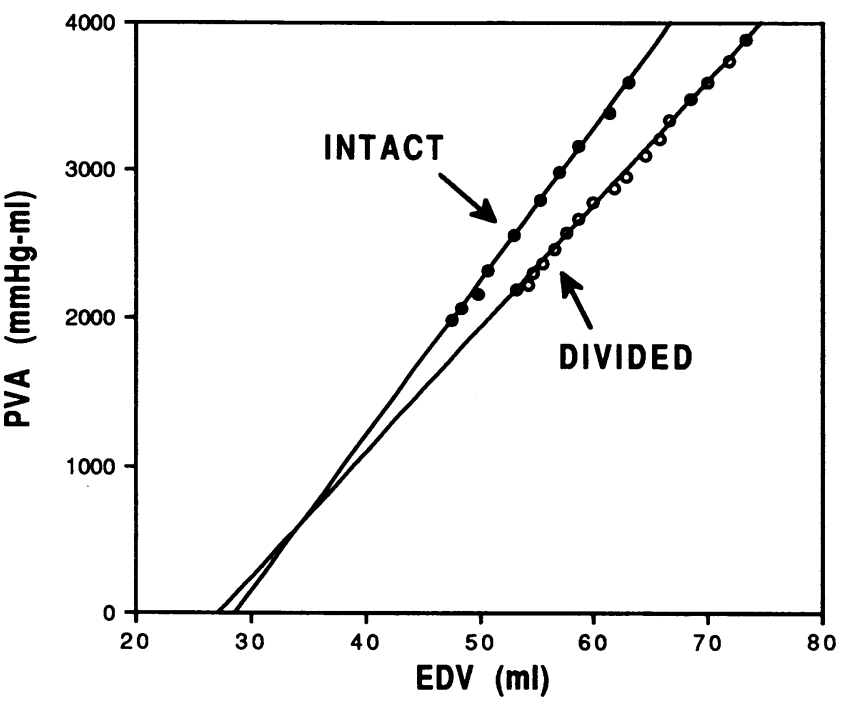

Figure 4. Representative pressure volume area-end-diastolic volume $(P V A-E D V)$ relations obtained during caval occlusion before (closed circles) and after (open circles) chordal division, which were linear within the physiologic pressure range. In each animal, there was a decline in the slope of the $P V A-E D V$ relationship, indicating reduced mechanical energy generated at an equivalent preload.

The integrity of the mitral subvalvular apparatus significantly influenced global LV systolic performance as reflected by a $72 \%$ decline in $E_{\text {es }}$, the slope of the linear ESPVR, after chordal severing. However, the extrapolated volume axis intercept decreased significantly into the (nonphysiologic) negative range. Reduced $V_{0}$, in theory, would suggest an increase in systolic function as a totally unloaded ventricle can shorten more when pump performance is enhanced; moreover, the linearly extrapolated $V_{0}$ tends to change in the same direction as $E_{\mathrm{es}}$ in response to inotropic interventions $(18,19)$. The data, therefore, can be interpreted as either nonlinearity of the ESPVR at high and low contractile states (12) or a change in the curvilinearity of the ESPVR, viz., a function of inotropic state $(11,12)$. In this study, a quadratic equation provided a significantly better fit of the end-systolic pressure volume points than did linear regression since the coefficient $a$ overall was significantly less than zero. After chordal division, $a$ was smaller (less negative) in all animals suggesting reduced concavity of the ESPVR with respect to the volume axis. This change attained only possible statistical significance, however, probably due to the high variability between individual dogs. $V_{0}$ was significantly lower, however, again reflecting the decrease in concavity of the curvilinear ESPVR with respect to the volume axis. The range of $V_{0}$, however, remained well within the physiologic range (vs. the $V_{0}$ values derived by linear regression [7]), supporting the appro-

Figure 3. Summary of the effects of chordal division on parameters characterizing nonlinear ESPVR and $P R S W$. Error bars represent \pm 1 standard deviation. (a) Coefficient of curvilinearity; $(b)$ slope of ESPVR at $V_{0} ; V_{0}$, volume axis intercept of the nonlinear ESPVR; $V_{100}$, end-systolic volume at end-systolic pressure of $100 \mathrm{mmHg} . M_{\mathrm{w}}$, slope of the stroke work-end-diastolic volume relationship; $V_{\mathrm{w}}$, $P R S W$ end-diastolic volume axis intercept; $V_{w 1,000}, P R S W$ enddiastolic volume at stroke work of $1,000 \mathrm{mmHg} \cdot \mathrm{ml}$. 

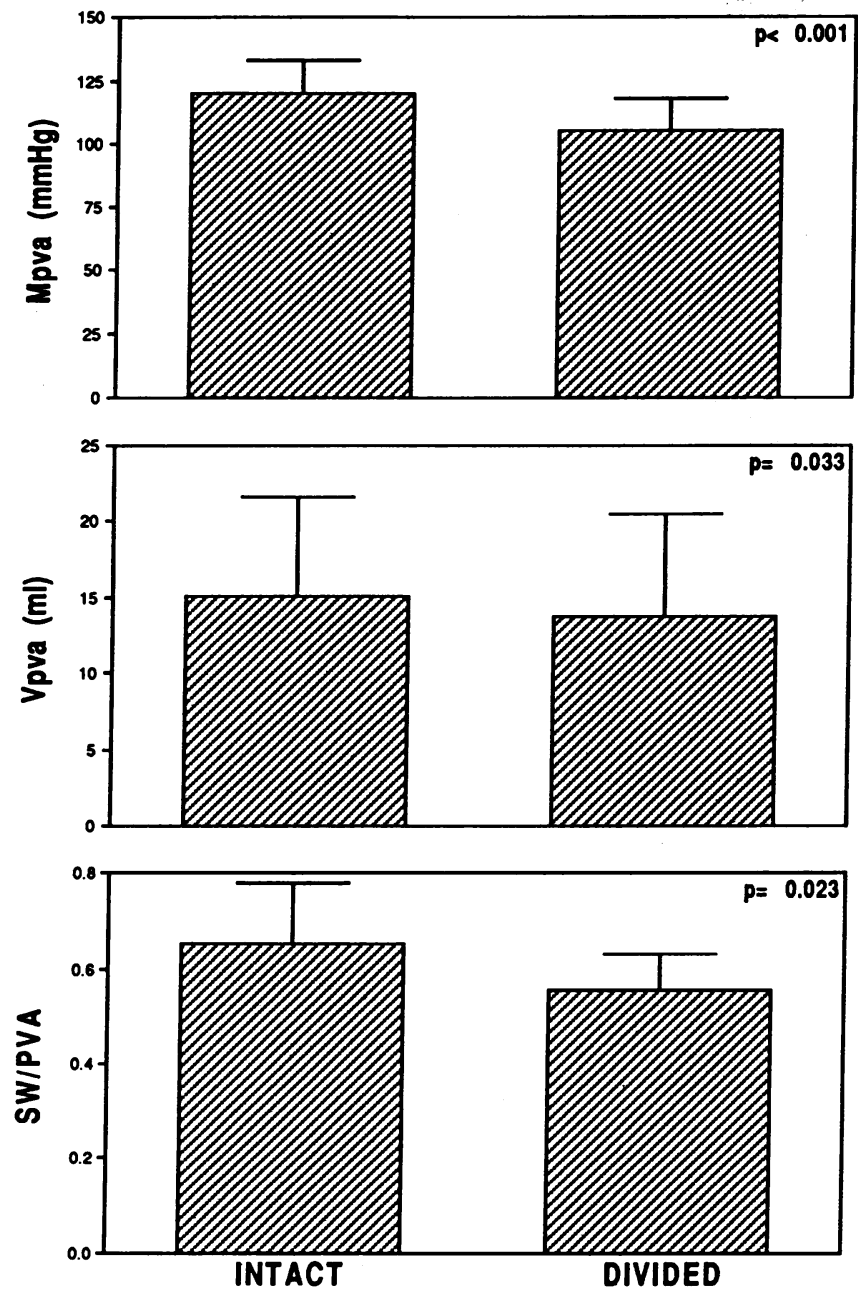

Figure 5. Summary of the effects of chordal division on LV energetics. Error bars represent \pm 1 standard deviation. $M_{\text {pva }}$, slope of the pressure volume area-end-diastolic volume $(P V A-E D V)$ relationship; $V_{\text {pva }}$, end-diastolic volume axis intercept; $S W / P V A$, efficiency of transfer of energy from $P V A$ to external stroke work at matched $E D V$.

priateness of a quadratic fit. The difference between $V_{0}$ obtained from quadratic and linear extrapolations may diverge further with impaired LV function (19). Thus, the negative values of the linearly extrapolated $V_{0}$ obtained after chordal severing by Sarris et al. (7) can best be explained by ESPVR nonlinearity. Since the coefficient $b$, the slope of the ESPVR at $V_{0}$, bears a parabolic relation to $a$ when examined over a wide range of contractile states (12), it was also used to assess LV pump mechanics. Chordal disruption resulted in a significant decline $(75 \%)$ in $b$, comparable to the relative magnitude of the fall in $E_{\text {es }}$ calculated previously using linear ESPVR (7), both of which indicate a deterioration of systolic $L V$ mechanical performance.

Alternatively, we also determined the shift of the ESPVR relation $\left(V_{100}\right)$ obtained from the linear fit of end-systolic pressure-volume points. Previously, the direction of shift of the linear ESPVR within the physiologic range has been directly associated with increased inotropy (18-23); i.e., positive and negative inotropic stimulation shifted the linear ESPVR upward to the left and downward to the right, respectively. This

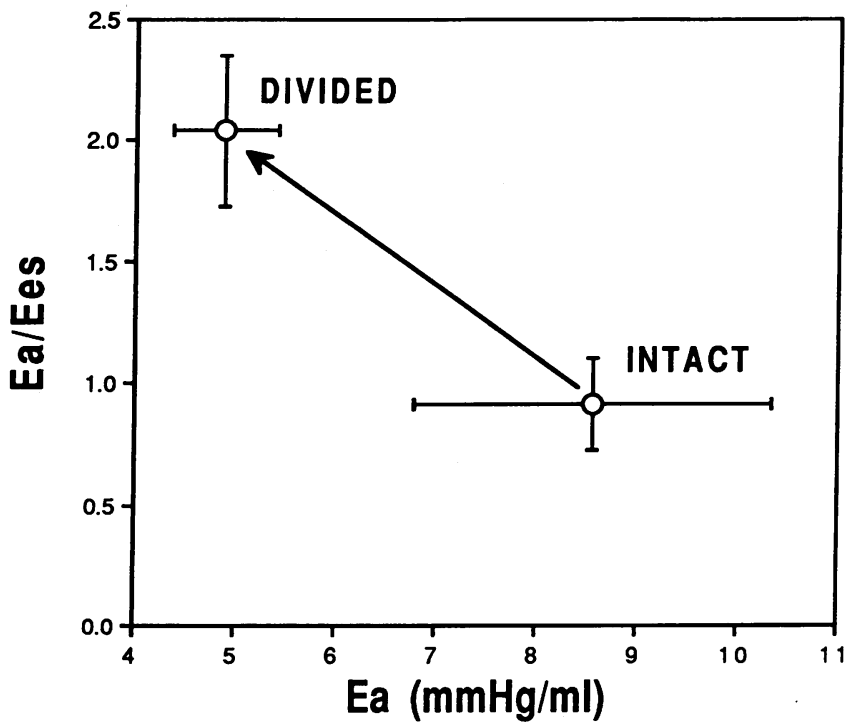

Figure 6. The effects of chordal division on $E_{\mathrm{a}}$ and the $E_{\mathrm{a}} / E_{\mathrm{es}}$ ratio. The increase in the $E_{\mathfrak{a}} / E_{\mathrm{es}}$ ratio indicates a mismatch between the left ventricle and the systemic arterial load. Error bars represent \pm 1 SEM.

method avoids extrapolation beyond the observed data range. Moreover, a similar value is calculated independent of the degree of polynomial fit (linear vs. quadratic). In this study, chordal division was associated with a $42 \%$ increase $(P=0.014)$ in $V_{100}$, indicating a larger $V_{\text {es }}$ at similar $P_{\text {es }}$; this shift of the linear ESPVR to the right suggests a reduction in LV systolic mechanics.

Because the coefficients of the quadratic fit are not entirely orthogonal (some degree of interdependence is present), $P R S W$ was also examined for corroboration. The $S W-E D V$

Table II. Effects of Chordal Division on LV Chamber Dimensions and Eccentricity at Matched LV End-diastolic Pressure

\begin{tabular}{lcc}
\hline & \multicolumn{2}{c}{ Chordal status } \\
\cline { 2 - 3 } & \multicolumn{1}{c}{ Intact } & Divided \\
\hline Base-apex axis $\left(D_{\mathrm{BA}}\right)$ & & \\
$\quad$ EDD $(\mathrm{mm})$ & $81.6 \pm 5.4$ & $84.8 \pm 5.4^{*}$ \\
EED $(\mathrm{mm})$ & $81.9 \pm 7.1$ & $85.0 \pm 7.1^{*}$ \\
dD $(\%)$ & $-0.1 \pm 2.9$ & $-0.1 \pm 3.4$ \\
Anterior-posterior axis $\left(D_{\mathrm{AP}}\right)$ & & \\
$\quad$ EDD $(\mathrm{mm})$ & $59.0 \pm 5.2$ & $60.1 \pm 4.6^{\ddagger}$ \\
EED $(\mathrm{mm})$ & $56.6 \pm 4.2$ & $57.1 \pm 3.8$ \\
dD $(\%)$ & $3.2 \pm 3.3$ & $4.7 \pm 2.7^{5}$ \\
Septal-lateral axis $\left(D_{\mathrm{SL}}\right)$ & & \\
$\quad$ EDD $(\mathrm{mm})$ & $45.7 \pm 3.8$ & $47.4 \pm 3.8^{5}$ \\
EED (mm) & $40.7 \pm 3.9$ & $40.9 \pm 1.1$ \\
dD $(\%)$ & $10.9 \pm 5.0$ & $13.8 \pm 5.0^{11}$ \\
End-diastolic eccentricity & & \\
$\quad$ index & $0.687 \pm 0.051$ & $0.701 \pm 0.051^{5}$ \\
End-ejection eccentricity & & \\
$\quad$ index & $0.718 \pm 0.017$ & $0.733 \pm 0.049^{\prime}$ \\
\hline
\end{tabular}

Vs. chordae intact: ${ }^{*} P<0.001 ;^{\ddagger} P=0.06 ;^{8} P<0.05 ;{ }^{\prime \prime} P=0.054 ;^{1} P$ $=0.067$. 
relation was highly linear; after chordal severing, the slope $\left(M_{\mathrm{w}}\right)$ decreased without any significant change in the volume axis intercept, indicating reduced global pump function. It should be noted, however, that $M_{\mathrm{w}}$ fell by only $14 \%$ compared with $39 \%$ reported earlier (7). This discordance may be explained by the different method of $S W$ calculation: $S W$ had been calculated from aortic flow measurements in our previous study by the equation: $S W=S V \cdot(M E P-E D P)$. Such external work based on aortic flow or cardiac output, however, does not fully describe the mechanical work performed by the ventricle since diastolic work may be inaccurately determined, particularly at high filling pressures. Given the absence of associated alterations in the end-diastolic pressure-volume relation after chordal division (7), the increase in $E D V$ would imply that the ventricle was operating in a higher range of end-diastolic pressures, thereby resulting in greater underestimation of $S W$ after chordal division. This may, in part, be responsible for the relatively greater reduction in $P R S W$ when aortic flow was used to compute $S W(7)$. Despite wide variation between animals, $V_{w 1,000}$ increased significantly by $17 \%$ after chordal division, indicating a greater preload for equivalent external work; this rightward shift of the $S W-E D V$ relation also implies deterioration in LV systolic pump function.

In the time-varying elastance model of the left ventricle, $P V A$ represents the total mechanical energy generated by each contraction (24-26). It consists of external mechanical work performed during systole and elastic potential energy stored in the myocardium at end-systole. Like $P R S W$, the $P V A-E D V$ relation was highly linear in this current experiment, and was unaffected by chordal severing. After disruption of the subvalvular apparatus, the slope of this relationship $\left(M_{\mathrm{pva}}\right)$ declined significantly by $13 \%$, thereby indicating reduced $P V A$ at similar LV preload $(E D V)$. Although statistically significant, the associated decrease in the volume intercept, $V_{\text {pva }}$, was small and probably of no major physiologic importance. $P V A$, however, is only an intermediary in the transfer of energy from myocardial $\mathrm{O}_{2}$ consumption $\left(M V \mathrm{O}_{2}\right)$ to external mechanical work. The efficiency of energy transfer in the final step of myocardial energy transfer from $P V A$ to $S W(S W / P V A)$ fell by $14 \%$ (at matched $E D V$ ) after the chordae were severed. $S W / P V A$ correlates inversely with the $E_{\mathrm{a}} / E_{\text {es }}$ ratio, and can be approximated by the equation (15):SW/PVA $=1 /\left[1+\left(0.5 E_{\mathrm{a}} / E_{\mathrm{es}}\right)\right]$. The overall cardiac mechanical efficiency of converting $\mathrm{O}_{2}$ to useful external work $\left(S W / M V \mathrm{O}_{2}\right)$, however, depends not only on $S W / P V A$, but also on $P V A / M V \mathrm{O}_{2}$. With a decline in $E_{\text {es }}$ after chordal division, one might anticipate a downward shift of the $M V \mathrm{O}_{2}-P V A$ relation to reflect decreased oxygen demand for excitation-contraction coupling (27), thereby increasing $P V A$ / $M V \mathrm{O}_{2}$ efficiency at any $P V A$ while maintaining a relatively constant $P V A$ /excess $M V \mathrm{O}_{2}$ efficiency. This is unlikely, however, since one would not expect chordal division to alter the metabolic state of cardiac myocytes. Moreover, there is clinical evidence that in patients with moderate heart failure mechanical efficiency is reduced, although not to the extent as $S W /$ $P V A$, presumably due to an increase in the $P V A / M V \mathrm{O}_{2}$ relationship (28). In contrast, in patients with severe heart failure who can no longer maintain adequate forward $S W$, there is a trend towards further reduction in $S W / M V \mathrm{O}_{2}$ due to a substantial fall in the $S W / P V A$ efficiency quotient. In either case, a decline of $S W / M V \mathrm{O}_{2}$ efficiency should accompany chordal division.
Recently, several studies have provided evidence that the left ventricle and the arterial circulation interact in such a manner as to optimize cardiac energetics (28-36), although whether external work $(S W)$ or mechanical efficiency $\left(S W / M V \mathrm{O}_{2}\right)$ is the critical factor is still a matter of debate. From the standpoint of $\mathrm{O}_{2}$ delivery to the peripheral tissue, optimization of $S W$ would be preferable, whereas optimization of efficiency is more appropriate in terms of cardiac energy expenditure. Based on data obtained from isolated canine heart experiments, Burkhoff and Sagawa (35) developed an analytical model relating $\mathrm{LV}$ energetics in terms of ventricular and arterial elastances. Corroborating the work of Sunagawa et al. (31), $S W$ was maximal when $E_{\mathrm{a}}$ equaled $E_{\mathrm{es}}$; however, $S W / M V \mathrm{O}_{2}$ was always maximal when $E_{\mathrm{a}}$ was approximately one-half of $E_{\text {es }}$ at any given $E D V(35)$. In this study, the $E_{\mathrm{a}} / E_{\text {es }}$ ratio was near unity at baseline $(0.91 \pm 0.53)$, suggesting that the ventricle and arterial properties were matched such that external stroke work was maximized; however, $E_{\mathrm{a}} / E_{\text {es }}$ was not statistically different from 0.5 . Thus, it was also possible that optimal $S W /$ $M V \mathrm{O}_{2}$ might have been the initial operating point during baseline conditions in this experiment. Although $E_{\mathrm{a}}$ fell significantly by $43 \%$ after chordal division, $E_{\mathrm{a}} / E_{\mathrm{es}}$ increased due to a proportionally greater $(-72 \%)$ reduction in $E_{\text {es }}$, indicating that the coupling between pump and load was altered such that neither $S W$ or $S W / M V O_{2}$ efficiency was optimal. Such is a characteristic of $\mathrm{LV}$ pump failure, as demonstrated in dog studies (34) and in patients with failing hearts (28). The effective arterial elastance, as defined by Sunagawa (37), has the dimension of volume elastance (i.e., $\mathrm{mmHg} / \mathrm{ml}$ ). It depends not only on the material properties of the arterial tree, but also on the systemic arterial pressure during ejection. As defined, $E_{\mathrm{a}}$ reflects the ratio of $P_{\text {es }}$ to $S V$ (37). After chordal division, $P_{\text {es }}$ was reduced substantially, as was mean ejection pressure, without any significant change in stroke volume, thereby resulting in a decrease in $E_{\mathrm{a}}$. Although only possibly significant statistically, derived total peripheral resistance fell (as no detectable changes in cardiac output occurred after chordal severing), which was probably chiefly responsible for the decline in $E_{\mathrm{a}}$. Whether this was mediated through direct peripheral $\beta_{2}$ adrenergic stimulation or via other mechanical reflex responses is not known. It is also possible that the significant, albeit small, decrease in heart rate could have contributed to the fall in $E_{\mathrm{a}}$ since effective arterial elastance is inversely related to the cardiac cycle length (37). This is unlikely, however, given the neglible change (6 $\left.\mathrm{min}^{-1}\right)$ in heart rate. The physiologic reason for the decline in $E_{\mathrm{a}}$ is not understood. Since ventriculo-arterial coupling was initially set such that external pressure-volume work was maximal during baseline conditions (i.e., $E_{\mathrm{a}} / E_{\mathrm{es}} \approx 1$ ), it was possible that in the setting of acute chordal division the lower $E_{\mathrm{a}}$ might reflect some feedback mechanism by which adequate forward cardiac flow output was maintained during moderate heart failure (28). Reduced $E_{\text {es }}$ accompanying LV failure also implies that the ventricle operates more as a pressure pump (small changes in pressure affect a proportionally larger change in stroke volume) since conservation of adequate systemic pressure takes precedence over systemic flow output; this interplay may thereby limit the extent of any potential compensatory decrease in $E_{\mathrm{a}}$. The absolute value of $S W / M V \mathrm{O}_{2}$ efficiency after chordal division, however, might have been compensated for by the observed increase in LV EDV (7), which has a direct, sigmoidal relation to ventricular efficiency (35). 
Although the mechanism responsible for valvular-ventricular interaction remains unknown, it should be emphasized that the deterioration of LV systolic mechanics after chordal division, as reflected by declines in these relatively load-independent indices of $\mathrm{LV}$ performance, was not due to a change in the intrinsic myocardial contractile state per se, as no myocardial injury occurred. More likely, the decline in LV systolic function resulted from perturbation of the normal three-dimensional geometry of the ventricle. Despite increases in EDD in all three axes after chordal division, the LV chamber became more elliptical at end-diastole. This was due to a relatively greater increment in the LV major axis, as previously reported $(38,39)$. The small, albeit insignificant, increase in stroke volume after chordal severing, however, was due primarily to augmented fractional dimensional shortening along the two minor LV dimensions. Thus, this increase in chamber eccentricity was possibly present $(P=0.067)$ also at end-ejection. Previously, Rushmer had observed sphericalization of the canine heart in early systole during isovolumic contraction with an abrupt shortening of the major axis and expansion of the minor axes (40). He attributed this phenomenon to the earlier activation and contraction of the papillary muscles, thereby resulting in the descent of the mitral annulus toward the ventricular apex. This initial stretch of the circumferential LV midwall fibers was postulated to enhance LV systolic performance via the Frank-Starling mechanism. We, therefore, hypothesize that the changes in LV geometry associated with disruption of the mitral subvalvular apparatus represents a form of mechanical disadvantage, which leads to the deterioration in LV systolic mechanics and reduced $S W / P V A$ efficiency. To our knowledge, this is the first demonstration that a change in LV geometry alone without an alteration in myocardial contractile state can have deleterious effects on LV energetics and ventriculo-arterial coupling.

Several potential limitations of this analysis should be discussed. First, in an attempt to minimize the possibility of overestimating $P V A$ (and thereby underestimating $S W / P V A$ ), $V_{0}$ derived from the quadratic fit of the ESPVR data was used to calculate $P V A$. The volume axis intercept originally used by Suga, however, was that obtained directly from measurement of the linear ESPVR (24-26). Since it was conceivable that our conclusions may have been different if PVA had been computed according to Suga's original definition, $\mathrm{LV}$ energetic analysis was repeated using the $V_{0}$ values derived from the linear ESPVR. Although the absolute values differed, the general results were similar: After the chordae were severed, the slope of the $P V A-E D V$ relationship declined by $6 \%(124 \pm 10$ vs. $116 \pm 15 \mathrm{mmHg}, P=0.024)$ with a leftward shift of $V_{\mathrm{pva}}$ $(13.7 \pm 5.6$ vs. $6.8 \pm 6.1 \mathrm{ml}, P=0.001)$. Similarly, $S W / P V A$ at matched LV EDV decreased with chordal division $(0.60 \pm 0.14$ vs. $0.38 \pm 0.06 \%, P=0.001)$. The magnitude of this average decline, however, was substantially greater than that computed from the curvilinear $V_{0}$ analysis $(35.1 \pm 12.1$ for the linear model vs. $13.7 \pm 11.4 \%$ for nonlinear, $P<0.001)$. This is consistent with the hypothesis that linearly extrapolated $V_{0}$ can shift into the negative volume range with chordal severing, thereby artifactually exaggerating $P V A$ and underestimating $S W / P V A$. Second, autonomic reflexes might have contributed to the changes observed in this study (despite the theoretical scientific merit of autonomic blockade, we felt this was not realistic in the immediate postcardiopulmonary bypass setting). This was unlikely, however, since both heart rate and total peripheral impedance did not increase despite the deterioration in LV performance after chordal division. This was probably due to the high adrenergic stimulation associated with cardiopulmonary bypass resulting from catecholamine release even though the animals were anesthetized (41). It is possible that the small decrease in heart rate may even reflect some vagal stimulation that could contribute to the deterioration of LV systolic function after chordal division. Again, the physiologic significance of this is unclear given such a neglible change. Third, although we have demonstrated a change in global LV chamber shape, the effect of chordal division on local wall geometry was not assessed. A final potential limitation is the use of a single, steady-state cardiac cycle to estimate $E_{\text {a }}$. Although instantaneous ventriculo-vascular matching can be more accurately determined by computing $E_{\mathrm{a}}$ for each beat during caval occlusion, this measurement may not actually represent actual effective arterial impedance (37). Furthermore, the steady-state ventriculo-arterial interaction is usually considered to have more clinical relevance.

In summary, the current analysis substantiates the validity of the concept of valvular-ventricular interaction. Chordal division resulted in deterioration of $L V$ systolic performance and an increase in the $E_{\mathrm{a}} / E_{\text {es }}$ ratio (because of a proportionally greater decline in $E_{\text {es }}$ compared with $E_{\mathrm{a}}$ ). This in turn led to a fall in the $S W / P V A$ (and, probably, $S W / M V \mathrm{O}_{2}$ ) efficiency. The integrity of the mitral subvalvular apparatus is, therefore, important not only in terms of global LV pump function, but also in terms of optimization of LV energetics via matching of the left ventricle to the effective arterial load. Although the actual mechanisms responsible for valvular-ventricular interaction have not been elucidated, they may be related to altered three-dimensional LV geometry, and systolic synergy of contraction. These data provide further support to the continuing clinical efforts to preserve as much of the subvalvular structures as possible during mitral valve surgery.

\section{Acknowledgments}

We thank the Baxter-Edwards Cardiovascular Surgery Division (Santa Ana, CA) for donating the bioprosthetic valves used in this study. We also thank Mitchell H. Sokoloff and Julie R. Glasson for their important technical help.

Supported by grant HL-29589 from the National Heart, Lung, and Blood Institute and the Veterans Administration Medical Research Service. Drs. Yun, Sarris, and Fann are Carl and Leah McConnell Cardiovascular Surgical Research Fellows.

\section{References}

1. Hansen, D. E., P. D. Cahill, W. M. DeCampli, D. C. Harrison, G. C. Derby, R. S. Mitchell, and D. C. Miller. 1986. Valvular-ventricular interaction: importance of the mitral apparatus in canine left ventricular systolic performance. Circulation. 73:1310-1320.

2. Spence, P. A., C. M. Peniston, T. E. David, N. Mihic, A. K. Jabr, P. Narini, and T. A. Salerno. 1986. Toward a better understanding of the etiology of left ventricular dysfunction after mitral valve replacement: an experimental study with possible clinical implications. Ann. Thorac. Surg. 41:363-371.

3. Spence, P. A. C. M. Peniston, N. Mihic, T. E. David, A. K Jabr, D. Archer, and T. A. Salerno. 1986. A physiological approach to surgery for acute rupture of the papillary muscle. Ann. Thorac. Surg. 42:27-30.

4. Hansen, D. E., P. D. Cahill, G. C. Derby, and D. C. Miller. 1987. Relative contributions of the anterior and posterior mitral chordae tendineae to canine global left ventricular systolic function. J. Thorac. Cardiovasc. Surg. 93:45-55.

5. Sarris, G. E., P. D. Cahill, D. E. Hansen, G. C. Derby, and D. C. Miller. 
1988. Restoration of left ventricular systolic performance after reattachment of the mitral chordae tendineae. J. Thorac. Cardiovasc. Surg. 95:969-979.

6. Hansen, D. E., G. E. Sarris, M. A. Niczyporuk, G. C. Derby, P. D. Cahill, and D. C. Miller. 1989. Physiologic role of the mitral apparatus in left ventricular regional mechanics, contraction synergy, and global systolic performance. $J$. Thorac. Cardiovasc. Surg. 95:527-533.

7. Sarris, G. E., J. I. Fann, M. A. Niczyporuk, G. C. Derby, C. E. Handen, and D. C. Miller. 1989. Global and regional left ventricular systolic performance in the in situ ejecting canine heart: importance of the mitral apparatus. Circulation. 80(Suppl. I):I-24-I-42.

8. Freeman, G. L. 1990. Improved cardiac performance secondary to dobutamine: the role of ventricular-vascular coupling. J. Am. Coll. Cardiol. 15:11361137.

9. Kono, A., W. L. Maughan, K. Sunagawa, K. Hamilton, K. Sagawa, and M. L. Weisfeldt. 1984. The use of left ventricular end-ejection pressure and peak pressure in the estimation of the end-systolic pressure volume relationship. Circulation. 70:1057-1065.

10. Alyono, D., V. E. Larson, and R. W. Anderson. 1985. Defining end-systole for end-systolic pressure-volume ratio. J. Surg. Res. 39:344-350.

11. Burkhoff, D., S. Sugiura, D. T. Yue, and K. Sagawa. 1987. Contractilitydependent curvilinearity of end-systolic pressure-volume relations. Am. J. Physiol. 252:H1218-H1227.

12. Kass, D. A., R. Beyar, E. Lankford, M. Heard, W. L. Maughan, and K. Sagawa. 1989. Influence of contractile state on curvilinearity of in situ end-systolic pressure-volume relations. Circulation. 79:167-178.

13. Glower, D. D., J. A. Spratt, N. D. Snow, J. S. Kabas, J. W. Davis, C. O. Olsen, G. S. Tyson, D. C. Sabiston, Jr., and J. S. Rankin. 1985. Linearity of the Frank-Starling relationship in the intact heart: the concept of preload recruitable stroke work. Circulation. 71:994-1009.

14. Goto, Y., B. K. Slinker, and M. M. LeWinter. 1990. Decreased contractile efficiency and increased nonmechanical energy cost in hyperthroid rabbit heart: relation between $\mathrm{O}_{2}$ consumption and systolic pressure-volume area or forcetime integral. Circ. Res. 66:999-1011.

15. Nozawa, T., Y. Yasumura, S. Futaki, N. Tanaka, M. Uenishi, and H. Suga. 1988. Efficiency of energy transfer from pressure-volume area to external mechanical work increases with contractile state and decreases with afterload in the left ventricle of the anesthetized closed-chest dog. Circulation. 77:1116-1124.

16. Sunagawa, K., W. L. Maughan, D. Burkhoff, and K. Sagawa. 1983. Lef ventricular interaction with arterial load studied in isolated canine ventricle. Am. J. Physiol. 245:H773-H780.

17. Rankin, J. S., P. A. McHale, C. E. Arentzen, D. Ling, J. C. Greenfield, and R. W. Anderson. 1976. The three-dimensional dynamic geometry of the left ventricle in the conscious dog. Circ. Res. 39:304-314.

18. Spratt, J. A., G. S. Tyson, D. D. Glower, J. W. Davis, L. H. Muhlbaier, C. O. Olsen, and J. S. Rankin. 1987. The end-systolic pressure-volume relationship in conscious dogs. Circulation. 75:1295-1309.

19. Little, W. C., C. P. Cheng, T. Peterson, and J. Vinten-Johansen. 1988. Response of the left ventricular end-systolic pressure-volume relation in conscious dogs to a wide range of contractile states. Circulation. 78:736-745.

20. Sodums, M. T., F. R. Badke, M. R. Starling, W. C. Little, and R. A. O'Rourke. 1984. Evaluation of left ventricular contractile performance utilizing end-systolic pressure-volume relationships in conscious dogs. Circ. Res. 54:731739.

21. Little, W. C. 1985 . The left ventricular $\mathrm{dP} / \mathrm{dt}_{\max }$-end-diastolic volume relation in closed chest dogs. Circ. Res. 56:808-815.
22. Little, W. C., A. Rassi, Jr., and G. L. Freeman. 1987. Effect of dobutamine and ouabain on left ventricular contraction and relaxation in closed-chest dogs. $J$. Clin. Invest. 80:613-620.

23. Crottogini, A. J., P. Willshaw, J. G. Barra, R. Armentano, E. I. Caberera Fisher, and R. H. Pichel. 1987. Inconsistency of the slope and the volume intercept of the end-systolic pressure-volume relationship as individual indexes of inotropic state in conscious dogs: presentation of an index combining both variables. Circulation. 76:1115-1126.

24. Suga, H. 1979. Total mechanical energy of a ventricle model and cardiac oxygen consumption. Am. J. Physiol. 236:H498-H505.

25. Suga, H., T. Hayashi, M. Shirahata, and I. Ninomiya. 1980. Critical evaluation of left ventricular systolic pressure volume area as predictor of oxygen consumption rate. Jpn. J. Physiol. 30:907-919.

26. Suga, H., R. Hisano, and I. Ninomiya. 1982. Digital on-line computation of a predictor of cardiac oxygen consumption: left ventricular systolic pressure volume area. Jpn. Heart J. 23:749-758.

27. Suga, H., Y. Goto, Y. Yasumura, T. Nozawa, S. Futaki, N. Tanaka, and M. Uenishi. 1988. $O$, consumption of dog heart under decreased coronary perfusion and propranolol. Am. J. Physiol. 254:H292-H303.

28. Asanoi, H., S. Sasayama, and T. Kameyama. 1989. Ventriculoarterial coupling in normal and failing heart in humans. Circ. Res. 65:483-493.

29. Elzinga, G., H. Piene, and J. P. de Jong. 1980. Left and right ventricular pump function and consequences of having two pumps in one heart: a study on the isolated cat heart. Circ. Res. 46:564-574.

30. Piene, $H_{\text {. }}$ and T. Sund. 1982. Does normal pulmonary impedance constitute the optimum load for the right ventricle? Am. J. Physiol. 242:H154-H160.

31. Sunagawa, K., W. L. Maughan, and K. Sagawa. 1985. Optimal arterial resistance for the maximal stroke work studied in isolated canine left ventricle. Circ. Res. 56:586-595.

32. van den Horn, G. J., N. Westerhof, and G. Elzinga. 1985. Optimal power generation by the left ventricle: a study in the anesthetized open thorax cat. Circ. Res. 56:252-261.

33. van den Horn, G. J., N. Westerhof, and G. Elzinga. 1986. Feline left ventricle does not always operate at optimum power output. Am. J. Physiol. 250:H961-H967.

34. Myhre, E. S. P., A. Johansen, J. Bjornstad, and H. Piene. 1986. The effect of contractility and preload on matching between the canine left ventricle and afterload. Circulation. 73:161-171.

35. Burkhoff, D., and K. Sagawa. 1986. Ventricular efficiency predicted by an analytical model. Am. J. Physiol. 250:R1021-R1027.

36. Toorop, G. P., G. J. van den Horn, G. Elzinga, and N. Westerhof. 1988 Match between feline left ventricle and arterial load: optimal external power or efficiency. Am. J. Physiol. 254:H279-H285.

37. Sunagawa, K., K. Sagawa, and W. L. Maughan. 1984. Ventricular interaction with the loading system. Ann. Biomed. Eng. 12:163-189.

38. March, R. J., K. A. Waters, T. Talbot, M. S. Visner, M. Jones, and R. E. Jones. 1986. Influence of native mitral apparatus on three-dimensional left ventricular geometry following mitral valve replacement. Surg. Forum. 17:306-308.

39. Gamms, E., W. Heimisch, S. Hagl, N. Mendler, H. Schad, and F. Sebening. 1987. Significance of the subvalvular apparatus following mitral valve replacement. Circulation. 76(Suppl. IV):IV-538. (Abstr.)

40. Rushmer, R. 1956. Initial phase of ventricular systole: asynchronous contraction. Am. J. Physiol. 188:187-194.

41. Kirklin, J. W., and B. G. Barratt-Boyes. 1986. Hypothermia, circulatory arrest, and cardiopulmonary bypass. In Cardiac Surgery. John Wiley and Sons, Inc., New York. 44-59. 